\title{
Decreases in COVID-19 are Related to Levels of Vaccination in 2947 Counties in the US
}

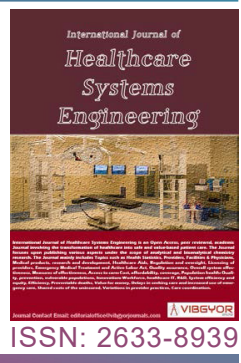

\section{Roger Craine*}

\section{Professor Emeritus, Economics, University of California, Berkeley, USA}

This note presents empirical evidence which supports the conventional wisdom that increases in vaccinations decrease the number of new COVID infections. A $1 \%$ increase in the percent of residents in a county that are vaccinated reduces the number of new infections in that county by roughly $1 \%$. My results contradict the conclusion of SV Subramanian and Akhil Kurmar (S\&K hereafter) boldly stated in the title "Increases in COVID-19 are unrelated to levels of vaccination across 68 countries and 2947 counties in the US" of their paper in Eur J Epidemiol, 2021 Sept 30: 1-4 [1]. The S\&K paper, the "Harvard study" is very popular with the anti-vax crowd [2].

I don't dispute S\&K's results. I disagree with their measure of policy effectiveness for the US. They use the weekly change in the daily (sevenday moving average of new daily) infections. The level of new infections is a better metric to judge effective policy. The weekly change in new COVID cases might be positive in counties (countries) that have a very effective COVID policy. For example, the weekly change in New Zealand's daily (per million residents) increased by 23 from 10/19/2021 to $10 / 26 / 2021$ while the US weekly change for the same period fell by 7655a. But when one looks at the daily level of new infections per million residents New Zealand's is 88 vrs the US at 73,695. New Zealand's policesb have been much more effective at protecting their residents from COVID infections than the US's.

So the question is: Do vaccinations prevent new infections in the real world? We know that clinical tests demonstrated that they are incredibly effective (most over 90\%) in preventing infections. Are they effective in practice? Yes, vaccines help stop the spread of COVID. I look at COVID infections/100k population in 2947 counties in the US vrs the percent of the county population fully vaccinated (the data used by S\&K.) My results show that roughly for each $5 \%$ of the county residents that get fully vaccinated the incidence of new infections declines by $5 \%$. These results strongly support the conventional wisdom that the most effective way to control the pandemic is to get your residents vaccinated and support policieslike vaccine mandates-that move the population toward herd immunity.

\section{Analysis}

I examine the county data for the US. I don't examine the international data because vaccines are not readily available in many countries. The county data come from S\&K's "Supplementary Information" at the end of their paper.

The data on the levels of new daily infections vrs the percent of the county residents fully vaccinated clearly show that vaccines are effective

*Corresponding author: Roger Craine, Professor Emeritus, Economics, University of California, Berkeley, USA

Accepted: December 29, 2021; Published: December 31, 2021

Copyright: (C) 2022 Craine R. This is an open-access article distributed under the terms of the Creative Commons Attribution License, which permits unrestricted use, distribution, and reproduction in any medium, provided the original author and source are credited.

Craine. Int J Healthc Syst Eng 2022, 3:010

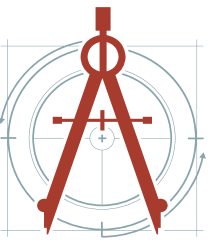

Citation: Craine R (2022) Decreases in COVID-19 are Related to Levels of Vaccination in 2947 Counties in the US. Int J Healthc Syst Eng 3:010 


\section{Cases/100K--Percent Vaccinated in County}

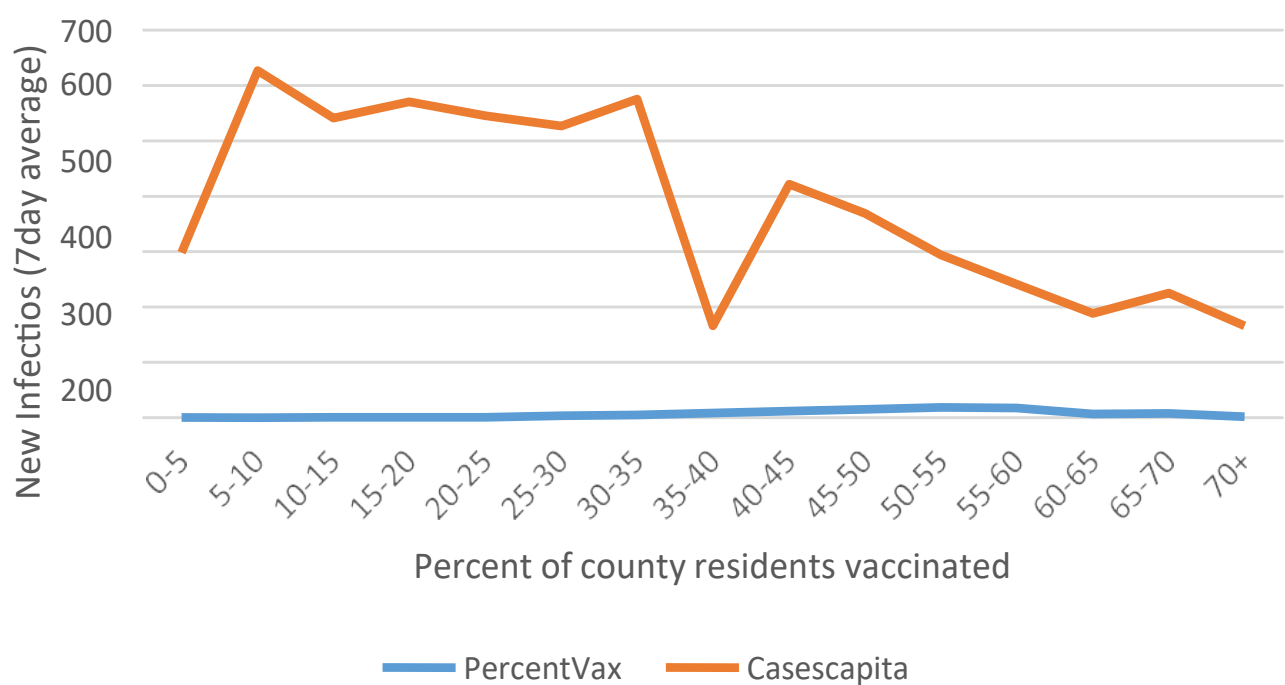

Figure 1: Vaccination percentage in each county and COVID infections.

Table 1: Regression statistics.

\begin{tabular}{|l|l|l|}
\hline & Slope & Intercept \\
\hline Coefficients & -5.45 & 601.96 \\
\hline Standard error & 1.44 & 65.51 \\
\hline P values & 0.0023 & 0.0000 \\
\hline $\mathbf{R}^{\wedge} \mathbf{2}$ & 0.52 & \\
\hline
\end{tabular}

in practice. A few counties have a low percent of their population vaccinated (5\% or less) and a low incidence of new COVID infections. These are rural counties with small populations. When you get to more representative counties with larger populations the level of new infections per $100 \mathrm{~K}$ residents jumps to a high plateau-600 to 500 new infections. New infections decline slightly as the percent of residents vaccinated in the counties increases from $10-35 \%$. Vaccinations help but are not enough to control the spread of infections. After you get to counties with greater than $40 \%$ of their residents vaccinated the new infection rate per $100 \mathrm{k} /$ population rapidly declines. The graph below shows the relationship between the percent of a county's residents that are vaccinated and new daily COVID infections (Figure 1).

To quantify the visual impression in the

\footnotetext{
atest data from Our World in Data.
}

${ }^{b}$ The new virulent Delta strain of COVID caused New Zealand to add vaccinations to its COVID policy toolbox. Their goal is to vaccinate $90 \%$ of the population. graph I ran a simple least squares regression. The results show that a $5 \%$ increase the county residents vaccinated leads to a $5.5 \%$ decrease in new infections- roughly one for one. Increase the percent of residents vaccinated and the percent of newly infected residents goes down. Vaccines are effective in the real world (Table 1).

The regression results firmly reject the null hypothesis of no relationship between the percent of county residents vaccinated and new infections in that county. The probability that the slope coefficient is zero is only 0.0023 . And we know from the clinical trials that the causality runs from vaccinations to protection from infections. So there's no issue about identification or causation.

\section{Conclusions}

The data and results in this note confirm the conventional wisdom that the most effective policy to end the pandemic is to vaccinate a high percentage (like 90\%) of the residents.

\section{References}

1. Subramanian SV, Akhil Kumar (2021) Increases in COVID-19 are unrelated to levels of vaccination across 68 countries and 2947 counties in the US. Eur J Epidemiol 30: 1-4.

2. https://www.motherjones.com/politics/2021/10/ subramanian-harvard-covid-vaccines/ 